SUBJECT AREAS:

PSYCHOLOGY

ANIMAL BEHAVIOUR

Received

10 June 2014

Accepted

20 November 2014

Published

6 January 2015

Correspondence and requests for materials should be addressed to A.W. (aw2@standrews.ac.uk) \title{
resource donation in capuchin monkeys, chimpanzees and humans
}

\author{
Nicolas Claidière', Andrew Whiten', Mary C. Mareno², Emily J. E. Messer', Sarah F. Brosnan ${ }^{2,3}$, \\ Lydia M. Hopper ${ }^{2,3,4}$, Susan P. Lambeth², Steven J. Schapiro ${ }^{2,5}$ \& Nicola McGuigan ${ }^{6}$
}

${ }^{1}$ Centre for Social Learning and Cognitive Evolution, School of Psychology \& Neuroscience, University of St. Andrews, St. Andrews, KY16 9JP, UK, ${ }^{2}$ Michale E. Keeling Center for Comparative Medicine and Research, UT MD Anderson Cancer Center, Bastrop, TX USA, ${ }^{3}$ Department of Psychology and Language Research Center, Georgia State University, PO Box 5010, Atlanta, GA 303025010, USA, ${ }^{4}$ Lester E. Fisher Center for the Study \& Conservation of Apes, Lincoln Park Zoo, Chicago, IL 606 14, USA, ${ }^{5}$ Department of Experimental Medicine, University of Copenhagen, Copenhagen, Denmark, ${ }^{6}$ School of Life Sciences, Heriot Watt University, Edinburgh, EHI 4 4AS, UK.

Prosocial acts benefitting others are widespread amongst humans. By contrast, chimpanzees have failed to demonstrate such a disposition in several studies, leading some authors to conclude that the forms of prosociality studied evolved in humans since our common ancestry. However, similar prosocial behavior has since been documented in other primates, such as capuchin monkeys. Here, applying the same methodology to humans, chimpanzees, and capuchins, we provide evidence that all three species will display prosocial behavior, but only in certain conditions. Fundamental forms of prosociality were age-dependent in children, conditional on self-beneficial resource distributions even at age seven, and conditional on social or resource configurations in chimpanzees and capuchins. We provide the first evidence that experience of conspecific companions' prosocial behavior facilitates prosocial behavior in children and chimpanzees. Prosocial actions were manifested in all three species following rules of contingency that may reflect strategically adaptive responses.

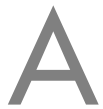

regard for the welfare of others is seen as one of the cardinal human virtues. Such prosocial dispositions show some cultural variation ${ }^{1,2}$, but humans often demonstrate extreme levels of selflessness, such as charitable contributions to anonymous strangers ${ }^{3}$. By contrast, several recent studies have concluded that such dispositions are lacking in our closest relatives, chimpanzees. In an influential experiment ${ }^{4}$, chimpanzee subjects could choose to pull either of two shelves, both of which delivered identical food items to themselves, but only one of which also delivered food to a second chimpanzee. Finding that the presence or absence of a social partner did not influence the choice chimpanzees made, the authors concluded that this form of prosocial resource donation is a unique disposition that has evolved in the human species since the split with the common chimpanzee/human ancestor (see also ref. 5).

Such a conclusion might be judged premature because the authors offered no comparative data demonstrating that children or other human participants would have behaved differently in their paradigm. There is also a range of experimental and observational evidence for other kinds of prosocial responses by chimpanzees ${ }^{6}$ in relation to familiar humans ${ }^{7}$ and to conspecifics ${ }^{8}$, both in captivity ${ }^{9}$ and the wild ${ }^{10,11}$. Moreover, when children were eventually tested in experiments using the aforementioned paradigm $\mathrm{m}^{4}$, young children, aged 3-4 years old, also failed to show prosocial responses ${ }^{12}$. Not until children were 7-8 years old did they do so; a striking discovery since replicated using a protocol based on the chimpanzee paradigm adapted for children ${ }^{13}$.

Such findings suggest prosociality of the kind identified in these studies might be shaped by years of experience of cultural practices in children's families and wider society. In turn this raises the question of whether prosocial behavior may be facilitated in non-human primates when they experience such behavior in their community. There is increasing evidence that non-human primate behavior can be profoundly changed by social learning ${ }^{14-16}$ and that cultural transmission shapes a particularly rich array of chimpanzee behavior patterns in the wild ${ }^{17,18}$, a conclusion supported by controlled experimental evidence for the transmission of multiple, short-term traditions in captive chimpanzee communities ${ }^{19-21}$. Although most of this evidence concerns foraging and tool use, there is observational evidence from the wild that social learning in primates can also affect social dispositions, such as 
lowered aggression ${ }^{22}$, and there is experimental evidence for such effects enhancing conciliatory dispositions after fights, and affiliative behavior more generally ${ }^{23,24}$. Taking this broad array of evidence concerning primate social learning into account, we designed what we believe are the first studies to test whether prosocial behavior in children, chimpanzees and capuchin monkeys may be shaped by experiences of prosocial behavior displayed by others.

We explicitly investigated this by first obtaining baseline data on prosocial responses of participants (human children and adults, chimpanzees, and capuchin monkeys), implementing the original method of Silk, et al. ${ }^{4}$ in as comparable a manner as possible; in a second phase, participants then experienced prosocial behavior by a new conspecific partner; finally, their own responses were then retested in a third phase with their original partner from phase one.

We considered two alternative ways in which participants might experience the prosocial behavior of others. In the 'triadic' case, as third parties, they would observe the prosocial behavior of one participant towards another; in the 'dyadic' case, they would themselves experience the effects of prosocial behavior by another individual. We had sufficient chimpanzee and capuchin participants available for only one of these and chose the dyadic alternative to test first, for two main reasons. First, we reasoned that directly benefitting from the prosocial behavior of another individual should provide the most powerful experience. If this is the case, this is the preferred option for this first experimental test of the influence of social learning on prosociality.

The second reason lay in relevance to recent theoretical analyses of the evolutionary roots of altruistic behavior. Altruistic behavior among unrelated individuals has long been understood as a viable outcome of repeated cycles of 'direct reciprocity' in which an altruistic act of individual $\mathrm{A}$ towards individual $\mathrm{B}$ is reciprocated by an altruistic act of B towards A, and so on. However in principle, altruism can be supported by 'indirect reciprocity', which in turn comes in two forms ${ }^{25}$. In one, having helped $\mathrm{B}, \mathrm{A}$ is more likely to be helped by others. This process, dependent on A's prosocial reputation being monitored by others, has been called 'downstream indirect reciprocity.' By contrast, in 'upstream reciprocity' $\mathrm{B}$ responds to an altruistic act received from A by becoming more likely to direct an altruistic act towards other individuals. This latter phenomenon was initially labeled 'generalized reciprocity $^{26}$. The scientific priority of this term ought perhaps to favor adopting it, but the contrast of 'upstream' versus 'downstream' indirect reciprocity is also instructive. We therefore favor preservation of both labels.

While at first, it is less easy to appreciate how upstream indirect reciprocity can be favored by natural selection compared to the downstream process, modeling analyses have demonstrated its theoretical viability ${ }^{25,27,28}$. However, despite its theoretical importance, only one empirical study (with rats) appears so far to have offered empirical data consistent with such a system ${ }^{28}$. Such results remain to be replicated in non-human species. Our experimental design addressed this because we arranged that in our experimental phase 2, each participant would experience prosocial acts directed towards itself by a conspecific, then, in phase 3 , be tested for enhanced prosocial responses towards the individual that they were paired with in the earlier baseline phase 1 .

Our major aims were therefore first, to study prosociality for three particularly relevant species in as comparable a fashion as possible, and second, to test the impact of experiencing prosocial behavior on later prosocial opportunities. We chose chimpanzees as our primary study species, because several studies have confirmed the lack of prosocial responses in varieties of the paradigm used by Silk et al. $^{5,29,30}$, yet there is evidence that chimpanzees may show strong social learning effects ${ }^{16,19,20}$ that might change these prosocial dispositions. Additionally, one chimpanzee study has recorded prosocial food donation in a related experimental design, although this involved a token exchange paradigm that bears less relationship to real-life scenarios in which food is directly offered ${ }^{31}$.

We included child samples for comparison, initially incorporating the age range of $4-8$ years, over which previous studies have indicated a significant change in the occurrence of prosocial resource donation $^{12,13}$. We attempted to apply to children as similar a paradigm to the chimpanzees' as possible, given limitations in earlier studies. In the first of these ${ }^{12}$, there had been no other child present when participant children made their choices (they were instead told that whatever was donated would be taken and given to another child). In the second ${ }^{13}$, the experiments were introduced as a 'game' and it appeared some children treated it as such, laughing when they withheld donation. The results we obtained with children led us additionally to include an adult human sample to provide a broader ontogenetic perspective.

We incorporated capuchin monkeys as a third subject population, because a prior study recorded a prosocial disposition using the twoshelves paradigm ${ }^{32}$, yet this was a small effect ( $\sim 65 \%$ prosocial choices against $50 \%$ by chance) and accordingly, the species is an apt candidate for testing effects of experiencing prosocial resource donation by others. Studies of other species of monkey have found some evidence of prosocial choices using variations on this paradigm $^{33}$.

In all three species, we incorporated into our Phase 1 baseline testing, species-appropriate variant conditions that would potentially influence the propensity to display prosocial responses. In children, we incorporated both a young age group ( 5 years) and an older one (7 years), predicting on the basis of prior research that prosociality would be seen only in the older group. Such age contrasts were not an option for the capuchin or chimpanzee samples available. For chimpanzees, the testing rooms offered the option to have pairs of individuals either separated or not. Predicting that the immediacy of the latter configuration might elicit prosocial responses hitherto reported as absent in this species, we contrasted these two configurations. Finally, we hypothesized that, as for marmosets ${ }^{34}$, food rewards appeared to be acting as a prepotent stimulus for capuchins, distracting subjects from making decisions that would or would not provision the other monkey, so we predicted that allowing monkeys to obtain their own reward, only after choosing between prosocial and non-prosocial options, might make the former choice more likely.

To first establish the level of prosociality of our participants in the baseline trials, we followed a protocol similar to Lakshminarayanan and Santos ${ }^{32}$. 'Proposer' and 'Receiver' individuals sat next to one another and faced a pair of shelves, vertically stacked and each baited with two rewards, one in front of each participant (Fig. 1). The Proposer--the focal experimental individual--had a choice of pulling one of two shelves, both of which delivered one reward to themselves and one to their test partner (the Receiver). Although both individuals always got a food item, the quality of the item varied. Depending on the shelf that the Proposer pulled, they either delivered a 'more preferred reward' (MPR - e.g. a high value food for the non-human primates) or a 'less preferred reward' (LPR - e.g. a low value food item; see Fig. 1) to their partner, the Receiver, whereas the two shelves on the Proposer's side always contained two identical rewards. In half the trials the Proposer's side held two MPRs and in the other half two LPRs.

Each Proposer participated in a counterbalanced series of trials presenting (i) 'Prosocial' options, where a Receiver could gain either a more preferred or a less preferred reward depending on which shelf the Proposer chose to pull; (ii) 'Empty control' trials with no Receiver present; or (iii) 'Self-centered' trials in which there was no Receiver present and the Proposer could reach across and take whichever reward they delivered on the Receiver's side. Delivering the MPR to the Receiver's side more in condition (i) than (ii) was taken to indicate a prosocial disposition, while (iii) served both to check that 

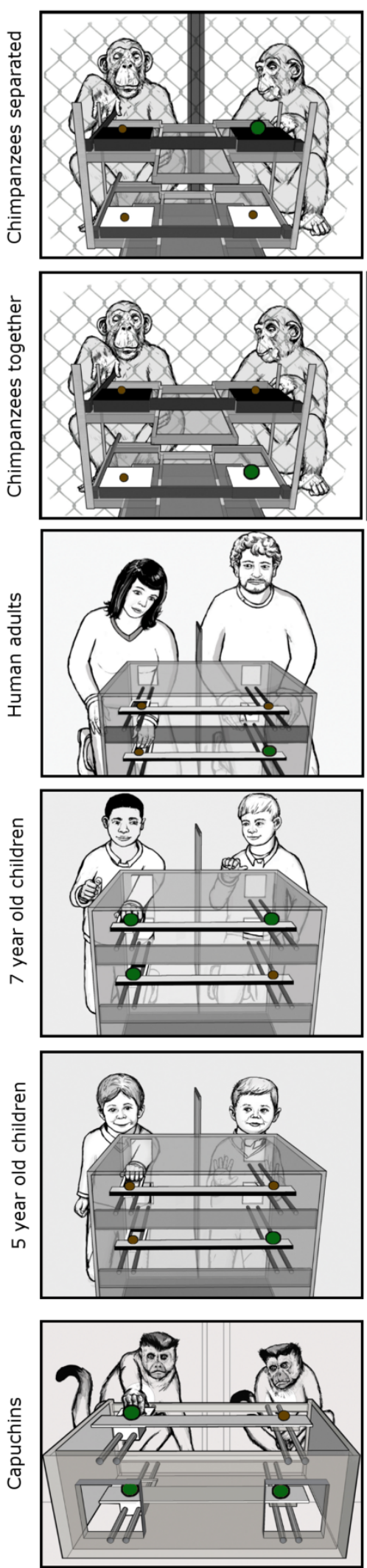

MPR

LPR

Empty Prosocial Self
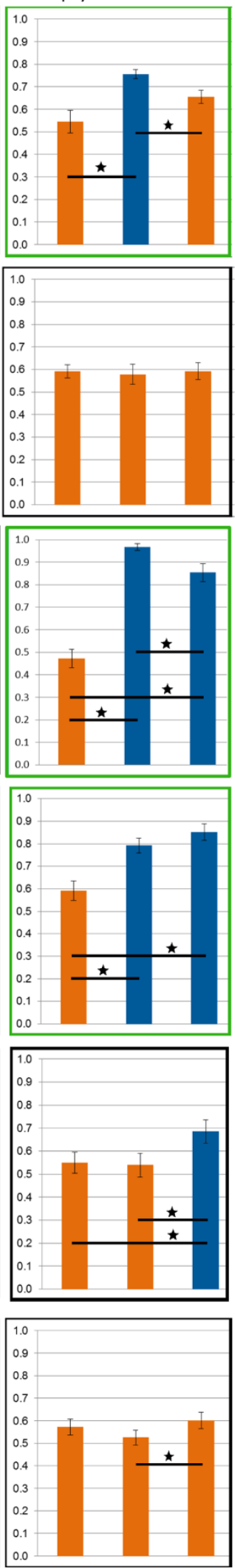

MPR

$E / S, x 2=3.34, d f=1, p=0.07$

$E / P, X 2=12.04, d f=1, p<0.001$

$P / S, X^{2}=5.95, d f=1, p=\mathbf{0 . 0 2}$

LPR:

$E / S, x 2=9.86, d f=1, p=\mathbf{0 . 0 0 1}$

$E / P, X 2=2.12, d f=1, p=0.15$

$P / S, X 2=5.51, d f=1, p=\mathbf{0 . 0 2}$
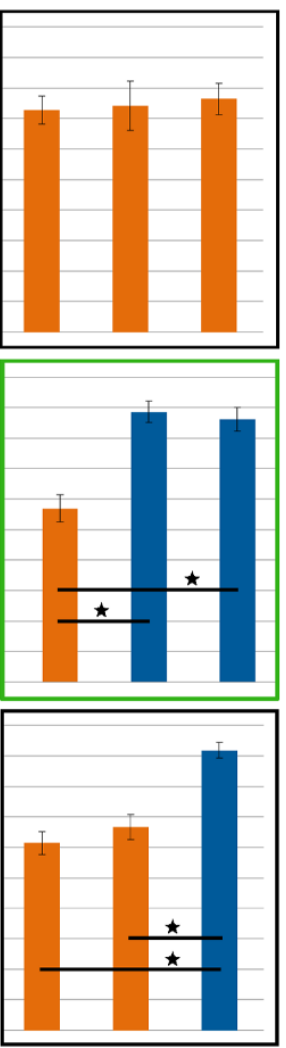

$E / S, x 2=0.00, d f=1, p=1.00$ $E / P, x 2=0.36, d f=1, p=0.55$ $P / S, X 2=0.06, d f=1, p=0.81$

LPR:

$E / S, x 2=0.61, d f=1, p=0.43$ $E / P, x 2=0.09, d f=1, p=0.76$ $P / S, X^{2}=0.11, d f=1, p=0.74$

MPR:

$E / S, x 2=35.97, d f=1, p<\mathbf{0 . 0 0 1}$

$E / P, x 2=51.09, d f=1, p<0.001$

$P / S, x 2=9.92, d f=1, p=\mathbf{0 . 0 0 1}$

LPR:

$E / S, x 2=20.49, d f=1, p<\mathbf{0 . 0 0 1}$

$E / P, X 2=22.89, d f=1, p<0.001$

$P / S, x 2=0.27, d f=1, p=0.60$

MPR:

$E / S, x 2=16.72, d f=1, p<0.001$

$E / P, X 2=12.58, d f=1, p<0.001$

$P / S, X 2=1.55, d f=1, p=0.21$

LPR:

$E / S, x 2=29.00, d f=1, p<0.001$

$E / P, X 2=0.87, d f=1, p=0.35$

$P / S, X 2=20.00, d f=1, p<0.001$

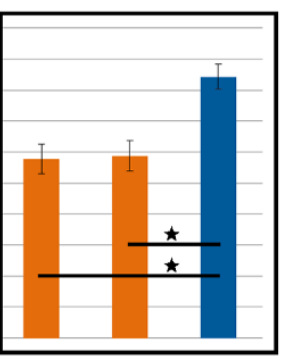

MPR:

$E / S, x 2=4.03, d f=1, p=0.05$

$E / P, X 2=0.03, d f=1, p=0.86$

$P / S, X 2=4.14, d f=1, p=0.04$

LPR:

$E / S, X 2=12.46, d f=1, p<0.001$

$E / P, X 2=0.02, d f=1, p=0.88$

$P / S, X 2=20.00, d f=1, p<0.001$

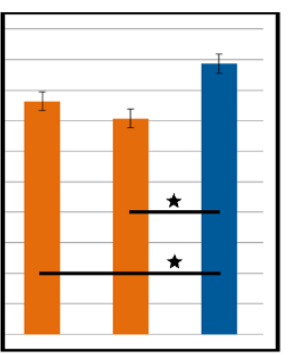

MPR:

$E / S, x 2=0.28, d f=1, p=0.60$

$E / P, x 2=0.59, d f=1, p=0.44$

$P / S, x 2=4.87, d f=1, p=0.03$

LPR:

$E / S, X 2=15.86, d f=1, p<0.001$

$E / P, X 2=1.74, d f=1, p=0.19$

$\mathrm{P} / \mathrm{S}, \mathrm{X} 2=12.27, \mathrm{df}=1, \mathrm{p}<\mathbf{0 . 0 0 1}$

Figure $1 \mid$ Prosocial responses of chimpanzees, humans and capuchins. The Y-axis represents the proportion of MPR pulled by the Proposer for the Receiver and the bars are marginal mean and standard deviation estimated from the statistical model. Graphs column 1, MPR for Proposer; column 2: LPR for Proposer. Empty (E) = Receiver absent; Prosocial $(\mathrm{P})=$ Receiver present; Self $(\mathrm{S})=$ Self-centered scenario, Receiver absent and Proposer access to Receiver compartment rewards. Blue chart bars = significantly different to Empty condition. Green-framed graphs indicate a significant prosocial response (Prosocial $>$ Empty). Drawings by Jason Zampol. 
the Proposer understood the consequences of their choice (e.g., that it delivered a reward to the Receiver's side) and to indicate the strength of any prosocial disposition compared to the 'self-centered' choices they made in this third condition (see Methods for further details).

After establishing the initial prosocial disposition of each Proposer-Receiver pair, participants were exposed in Phase 2 to a new partner who was trained to always choose the prosocial option. In Phase 3, participants were then re-tested using the same procedure and control conditions as in Phase 1 (see Methods for more details). The application of this procedure allowed us to measure the effect on the Proposer of exposure to a conspecific individual making prosocial choices and to compare that effect across species and, for humans, across developmental periods. It is important to note that any prosocial effects cannot be explained by direct reciprocation, because when re-tested as Proposers in Phase 3, participants were not partnered with the individual who had earlier acted prosocially towards them (in Phase 2).

In summary, we tested two broad sets of hypotheses. The first, addressed in the Phase 1 baseline trials, was whether prosociality was evidenced and varied according to the different conditions (age for the children; social and resource configurations for the chimpanzees and capuchins). The second concerned the effects of experiencing prosocial behavior in the intervention of Phase 2, assessed by comparing responses in the re-testing of Phase 3 with those in the baseline Phase 1.

\section{Results}

Developmental and evolutionary origins of prosociality. Results from the baseline phase of testing (Fig. 1) revealed conditional prosociality in all three species, but the conditionality took different forms. In humans, two factors affected the occurrence of prosocial behavior: age and relative reward magnitude (sex and friendship had small, non-significant effects; see Supplementary Information). Young children did not show significant prosocial tendencies: their tendency to deliver the desirable reward, the MPR, to the Receiver side was significantly greater in the selfcentered condition than in the empty and prosocial conditions. This was true both when the Proposer got the more- $\left(\chi^{2}=4.03\right.$, df $=1, \mathrm{p}=0.05$ and $\chi^{2}=4.14, \mathrm{df}=1, \mathrm{p}=0.04$ respectively) and lesspreferred rewards $\left(\chi^{2}=12.46, \mathrm{df}=1, \mathrm{p}=0.001\right.$ and $\chi^{2}=20.00, \mathrm{df}=$ $1, \mathrm{p}=0.001$ respectively). However, the Proposer's choices in the prosocial condition were not significantly different from their choices when the Receiver was absent (Empty control trials MPR: $\chi^{2}=0.03, \mathrm{df}=1, \mathrm{p}=0.86$; LPR: $\chi^{2}=0.02, \mathrm{df}=1, \mathrm{p}=0.88$; all comparisons are illustrated in Fig. 1). In contrast, the older children delivered the MPR to the Receiver's side more often in the prosocial than the empty condition when they themselves received the MPR $\left(\chi^{2}=12.58, \mathrm{df}=1, \mathrm{p}=0.001\right)$, but not when they received the LPR $\left(\chi^{2}=0.87, \mathrm{df}=1, \mathrm{p}=0.35\right)$. In this group, the Proposer's choices in the self-centered condition were also significantly different from those in the empty condition $\left(\chi^{2}=16.72, \mathrm{df}=1, \mathrm{p}=0.001\right)$, but not from those in the prosocial condition $\left(\chi^{2}=1.55\right.$, $\mathrm{df}=1, \mathrm{p}=$ $0.21)$ when the Proposer received the MPR, but were different from both when the Proposer received the $\operatorname{LPR}\left(\chi^{2}=29.00, \mathrm{df}=1, \mathrm{p}=\right.$ 0.001 and $\chi^{2}=20.00, \mathrm{df}=1, \mathrm{p}=0.001$ respectively). Finally, adults donated the MPR to the Receiver more in the prosocial than the empty condition, both when the Proposer received the MPR and the LPR $\left(\chi^{2}=51.09, \mathrm{df}=1, \mathrm{p}=0.001\right.$ and $\chi^{2}=22.89$, $\mathrm{df}=1$, $\mathrm{p}=0.001$ respectively). In both cases, choices in the self-centered condition were also significantly different from the empty condition (MPR: $\chi^{2}=35.97, \mathrm{df}=1, \mathrm{p}=0.001$ and LPR: $\chi^{2}=20.49, \mathrm{df}=1, \mathrm{p}=$ $0.001)$, whereas they differed from the prosocial condition only in the $\operatorname{MPR}\left(\chi^{2}=9.92, \mathrm{df}=1, \mathrm{p}=0.001\right), \operatorname{not} \operatorname{LPR}\left(\chi^{2}=0.27, \mathrm{df}=1, \mathrm{p}=\right.$ $0.60)$. These results show a progressive development of prosocial behavior, from its absence in young children, to its full expression in adults, such that adults gave their partner the more preferred reward, even when they themselves only received the less preferred reward.

Considering the chimpanzees, we found that Proposers chose the prosocial option only when both Proposer and Receiver had a barrier between them (the 'separated' condition) and so long as they also received a MPR themselves (like the older children). In the separated condition, chimpanzees chose the prosocial option more in the prosocial condition than the empty control when they received an MPR $\left(\chi^{2}=12.04, \mathrm{df}=1, \mathrm{p}=0.001\right)$, but not when they received an LPR $\left(\chi^{2}=2.12, \mathrm{df}=1, \mathrm{p}=0.15\right.$; all comparisons illustrated in Fig. 1). When the Proposer received an MPR, choices in the self-centered condition did not differ from the empty condition, but did from the prosocial condition $\left(\chi^{2}=3.34, \mathrm{df}=1, \mathrm{p}=0.07\right.$ and $\chi^{2}=5.95, \mathrm{df}=1$, $\mathrm{p}=0.02$ respectively), whereas in LPR it differed from both $\left(\chi^{2}=\right.$ 9.86, $\mathrm{df}=1, \mathrm{p}=0.001$ and $\chi^{2}=5.51, \mathrm{df}=1, \mathrm{p}=0.02$ respectively).

When chimpanzees were tested in the same enclosure, without a barrier between them, there were no differences in their responses across conditions (in MPR, empty vs. prosocial: $\chi^{2}=0.36$, $\mathrm{df}=1$, $\mathrm{p}=0.55$; empty vs. self-centered: $\chi^{2}=0.00, \mathrm{df}=1, \mathrm{p}=1.00$; prosocial vs. self-centered: $\chi^{2}=0.06, \mathrm{df}=1, \mathrm{p}=0.81$; in LPR, empty vs. prosocial: $\chi^{2}=0.09, \mathrm{df}=1, \mathrm{p}=0.76$; empty vs. self-centered: $\chi^{2}$ $=0.61, \mathrm{df}=1, \mathrm{p}=0.43$; prosocial vs. self-centered: $\chi^{2}=0.11, \mathrm{df}=1$, $\mathrm{p}=0.74)$.

Finally, capuchin Proposers showed no evidence of prosocial tendencies in the MPR or LPR conditions (other factors, such as age, sex and social proximity had no significant effects, see Supplementary Information). When receiving an MPR, Proposers' choices in the self-centered condition were different from those in the prosocial condition $\left(\chi^{2}=4.87, \mathrm{df}=1, \mathrm{p}=0.03\right)$, but not the empty condition $\left(\chi^{2}=0.28, \mathrm{df}=1, \mathrm{p}=0.60\right.$; all comparisons illustrated in Fig. 1). Choices in the prosocial and empty conditions did not differ $\left(\chi^{2}=\right.$ $0.59, \mathrm{df}=1, \mathrm{p}=0.44)$. When receiving an LPR, Proposers' choices in the self-centered condition differed from the empty and prosocial conditions $\left(\chi^{2}=15.86, \mathrm{df}=1, \mathrm{p}=0.001\right.$ and $\chi^{2}=12.27, \mathrm{df}=1, \mathrm{p}=$ 0.001 respectively), but there was no difference in choices between these latter two conditions $\left(\chi^{2}=1.74, \mathrm{df}=1, \mathrm{p}=0.19\right)$. Given that these results differed from those found in a previous study ${ }^{32}$ we decided to explore further this lack of prosociality by conducting a follow up study. We tested 3 hypotheses: (i) that a reduction of the difference between the MPR and LPR reward would make the capuchins less reluctant to give the MPR to the Receiver and thereby increase their prosocial tendencies; (ii) that the difference between the MPR and LPR on the Receiver's side was not well recognized and therefore that removing the LPR on the Receiver side would increase their prosocial tendencies; finally, (iii) that the presence of food facing the Proposer distracted him/her from the Receiver's side of the apparatus and that delaying the reward for the Proposer would increase their prosociality. However, we found evidence of a prosocial response in this species only when the capuchins received an MPR after pulling in a shelf (see Fig. 1 and Supplementary Information for more details on tests of hypothesis iii and for the results of tests of hypotheses $i$ and ii). Capuchins were more likely to deliver an MPR to the Receiver side in the self-centered condition (Mean $=.85, \mathrm{SE}=.054)$ compared to the prosocial condition (Mean $\left.=.63, \mathrm{SE}=.043, \chi^{2}=6.43, \mathrm{df}=1, \mathrm{p}=0.01\right)$ and to the empty condition $\left(\right.$ Mean $\left.=.55, \mathrm{SE}=.022 ; \chi^{2}=10.23, \mathrm{df}=1, \mathrm{p}=0.001\right)$. Delivery of the MPR to the Receiver was also significantly more frequent in the prosocial than the empty conditions $\left(\chi^{2}=4.20\right.$, $\mathrm{df}=1, \mathrm{p}=0.04)$.

Influence of social experience on prosociality. Results are illustrated in Fig. 2. Chimpanzees were significantly more likely to be prosocial after experiencing another chimpanzee consistently making prosocial choices. This was true both when the Proposer received an $\operatorname{MPR}\left(\chi^{2}=8.68, \mathrm{df}=1, \mathrm{p}=0.003\right)$ and an $\operatorname{LPR}\left(\chi^{2}=\right.$ 
7.08, $\mathrm{df}=1, \mathrm{p}=0.008)$. Comparatively, 7-year-old children showed increased prosociality, but only in the condition where the Proposer received an $\operatorname{MPR}\left(\chi^{2}=4.56, \mathrm{df}=1, \mathrm{p}=0.003\right)$, not when they received an $\operatorname{LPR}\left(\chi^{2}=0.30, \mathrm{df}=1, \mathrm{p}=0.59\right)$. Finally, there was no evidence that experiencing prosociality from another individual in either the MPR or LPR trials affected the responses of capuchin monkeys (MPR: $\chi^{2}=1.40, \mathrm{df}=1, \mathrm{p}=0.24$ and LPR: $\chi^{2}=0.93$, $\mathrm{df}=$ $1, \mathrm{p}=0.33$ ) or 5 -year-old children (MPR: $\chi^{2}=0.43, \mathrm{df}=1, \mathrm{p}=0.51$ and LPR: $\left.\chi^{2}=0.26, \mathrm{df}=1, \mathrm{p}=0.61\right)$. Given their baseline responses, adults did not experience Phases 2 and 3.

To test the possibility that the results of social experience emerged purely through an increase in delivery of the MPR to the Receiver side, we compared the results obtained during the first and second block of the first testing phase. A categorical variable representing the two blocks had a small and non-significant effect when added to the GEE with condition, reward, and their interaction. This was true for the capuchin monkeys $\left(\chi^{2}=0.69, \mathrm{df}=1, \mathrm{p}=.41\right)$ and the chimpanzees (separate condition, $\chi^{2}=0.45, \mathrm{df}=1, \mathrm{p}=0.50$; together condition, $\left.\chi^{2}=0.60, \mathrm{df}=1, \mathrm{p}=0.44\right)$. This result shows that the delivery of the MPR to the Receiver did not differ significantly between blocks across all three conditions (empty, prosocial and self-centered). Focusing only on the prosocial condition during the first testing phase, a GEE with only the block variable showed that the prosocial response of capuchins $\left(\chi^{2}=1.48, \mathrm{df}=1, \mathrm{p}=0.22\right)$ and chimpanzees (separate condition, $\chi^{2}=2.11, \mathrm{df}=1, \mathrm{p}=0.15$; together condition, $\chi^{2}=2.48, \mathrm{df}=1, \mathrm{p}=0.12$ ) did not differ significantly between blocks.

These results demonstrate a role for social experience in increasing prosocial behavior in older children and in our closest relative, the chimpanzee. Like the younger children, capuchins failed to show such an effect.

We also compared performance in the prosocial test condition to the two control conditions after the social learning phase (testing phase 2) using the same model as we used for the first testing phase (Fig. 3). The results confirm that only chimpanzees (most clearly in the LPR condition) and 7-year old children (most clearly in the MPR condition) responded to the social experience by becoming more prosocial.

\section{Discussion}

Phase 1 of our study revealed that members of all three species we studied may choose prosocial options in the social context we employed, but in each case such responses were conditional rather than universal. We discuss results for each species in turn and compare them, before addressing the results of the social experience intervention Phases 2 and 3.

The results for children indicate that prosocial responses in such donor scenarios are slow to develop. The younger children's failure to exhibit a prosocial tendency towards another child who was sitting close to them, and the older children's failure to do so if they themselves would obtain a less preferred reward, are striking demonstrations that even in our species, the emergence of such demanding forms of prosociality are late developing and somewhat fragile. Some elements of prosociality, including forms of helping and sharing behavior, have been identified in early childhood and infancy ${ }^{7,35-37}$, but our results are consistent with those of other recent studies suggesting that other aspects of prosocial behavior, such as the one we study here, are far from being fully developed even by the age of 7-8 years ${ }^{12,13,38}$. Only in adults did we find unconditional prosocial choices as the typical response, with a preferred reward being delivered to the Receiver even when the Proposer themselves gained only a less-preferred reward. We discuss this aspect further below because we found similar conditional effects in the chimpanzees as in the older children.

Our Phase 1 results for chimpanzees are the first to reveal prosocial responses in this paradigm, contrasting with the earlier studies that elicited no such effect ${ }^{4,5,29}$. Surprisingly, our positive results were obtained in the same primate center as the original study that found no prosocial disposition ${ }^{4}$. Perhaps the most obvious of the few differences in our methodologies was that, in our study, the Receiver could gain either a more preferred or a less preferred reward (as modeled on the capuchin study of Lakshminarayanan and Santos $^{32}$ ), as opposed to receiving a reward versus no-reward. This may have affected the Proposers' understanding of the consequences of their actions, although why the contrasts should be in this direction remains unclear. Another important difference is that we included a self-centered condition. This is important to establish understanding of the apparatus by the chimpanzees, yet was absent in the original study, making the results ambiguous. This could also explain why our findings are consistent with the more recent results of a modified version of this paradigm in which chimpanzees also expressed prosocial choices when choosing between tokens that could later be exchanged for food, rather than interacting with food items that were directly before them ${ }^{31}$.

The latter results may bear on the effect we found in both the older children and chimpanzees, where prosocial choices were made when the Proposer received the preferred reward (the MPR condition), but not when the Receiver would gain a preferred reward while the Proposer would gain only a less-preferred one (the LPR condition). A plausible explanation for this is an aversion to disadvantageous inequity; Proposers may have hesitated to make a choice in which the Receiver gained a more preferred reward than the Proposer ${ }^{33,39}$. It could be that the token-based approach of Horner, et al. ${ }^{31}$ mentally distanced participants from this consideration, whereas our approach allowed for an easy comparison. There is evidence for a sensitivity to disadvantageous inequity in a diversity of other experimental paradigms and contexts, in both chimpanzees and other nonhuman primates ${ }^{24,40}$, as well as in children, sometimes from an early age $^{10,41,42}$

However, inequity aversion could not have been too strong, or we would have expected to find that prosocial choices would be significantly lower in the LPR context than the $50 \%$ expected in the empty condition. We did not find such an effect in any of our analyses, for any species. The interesting difference between classic inequity aversion studies and our prosocial test that likely explains this is that in the former studies, the participant and another individual received the unequal resource offers from a third party, which can elicit strong protest and agonistic responses towards that party ${ }^{43}$. By contrast, in the LPR inequity condition of our dyadic prosocial paradigm, it is the Proposer who has direct control over whether or not it makes an offer that is to the advantage of the Receiver. Moreover the Proposer will get the same reward, whichever option it chooses. The lack of responses to inequity thus seems understandable and not in conflict with strong effects demonstrated in quite different contexts.

We also found a conditional expression of prosociality in our capuchin sample. Our Phase 1 results for capuchins did not reveal evidence of prosocial responses in this paradigm. However, our suspicion that the presence of food rewards in the direct gaze of Proposers acted as a prepotent stimulus, distracting them from potential prosocial choices, was confirmed by delaying the Proposer's reward until after they chose an option for the Receiver. In this context, prosocial choices emerged. These results are consistent with prior evidence of prosocial tendencies using this paradigm ${ }^{32}$, and more particularly, with the results of a study similar to that of Horner, et al. ${ }^{31}$, in which capuchins preferentially chose whichever of two tokens would be followed by rewards for both self and another capuchin sitting in an adjacent compartment, rather than a token that meant a reward only for self ${ }^{44}$.

Turning to the findings of the intervention of Phase 2, as tested in Phase 3, our results showed that social experience of prosocial behavior increased the prosocial responses of chimpanzees and sevenyear-old children who had not hitherto shown significant prosoci- 


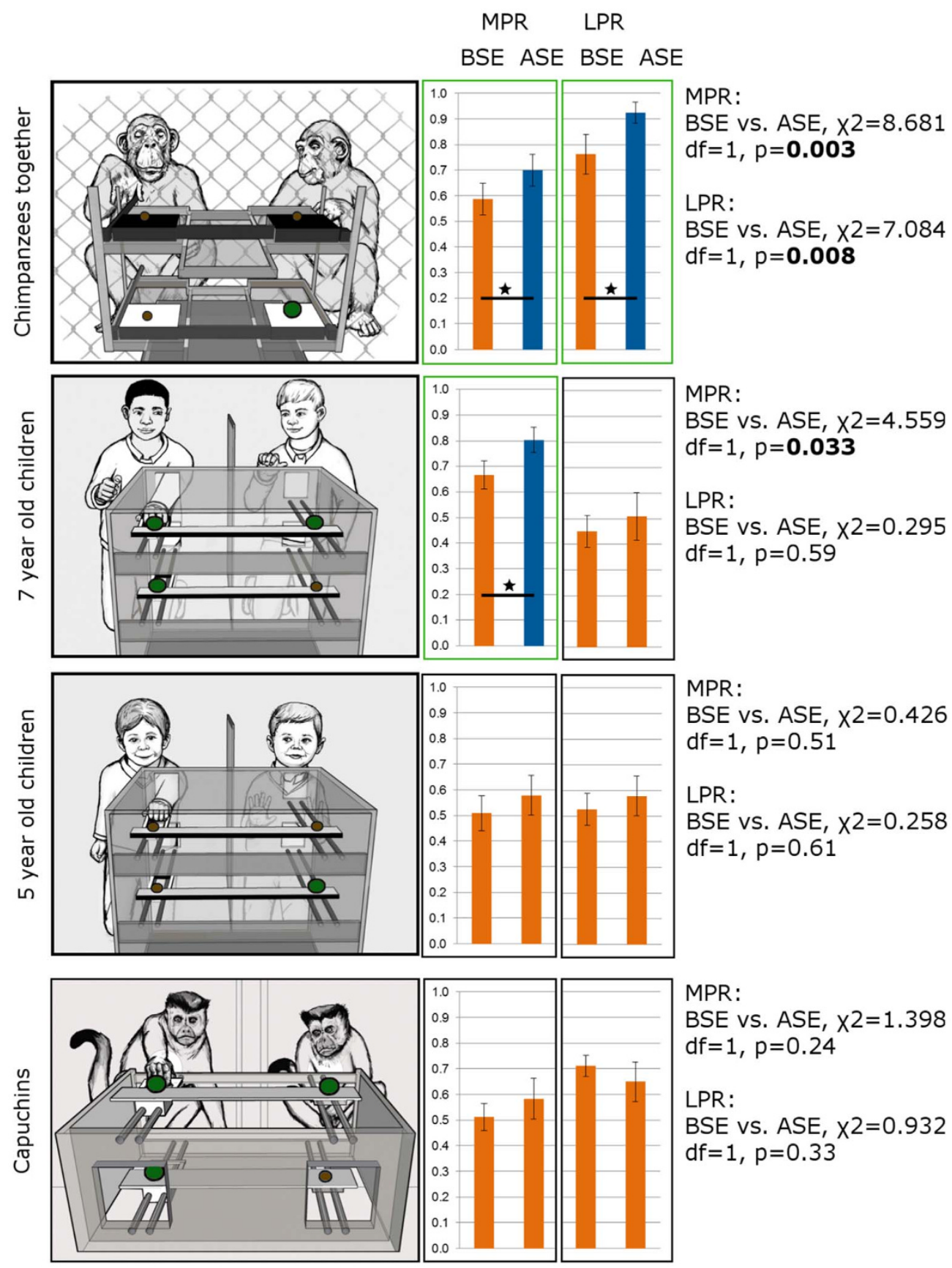

Figure $2 \mid$ Prosocial responses before and after social experience. The Y-axis represents the proportion of MPR pulled by the Proposer for the Receiver and the bars are marginal mean and standard deviation estimated from the statistical model. Graphs column 1, MPR for Proposer; column 2: LPR for Proposer. Before Social Experience (BSE) = prosocial response before exposure to the prosocial model; After Social Experience (ASE) = prosocial response after experience of a prosocial other. Blue chart bars = significantly different to BSE condition. Green-framed graphs indicate a significant increase in prosocial response (ASE $>$ BSE). Statistically significant differences are indicated by stars, with statistics on the right. Other conventions as for Fig. 1. Drawings by Jason Zampol.

ality. Interestingly, this effect was not seen in the younger children, suggesting that by seven years old, children either already display prosocial dispositions, or are otherwise at a stage of receptivity to social experiences in this domain. Those already acting prosocially may thus already have been shaped by such experiences in their everyday lives.

We believe we are the first to demonstrate these effects, which are consistent with two fundamental processes that tend to be examined in rather separate scientific literatures. First, our results meet the criteria of social learning, defined in a widely accepted way as "learning that is influenced by observation of, or interaction with, another animal (typically a conspecific) or its products"45. It is the second (interaction), rather than the first aspect (observation) in this definition that would be in play in our experiments: as noted in our introduction, a triadic context would be necessary for a subject to learn from observing the prosocial responses of a second individual towards a third, whereas our subjects must have learned directly from the behavior of another individual towards them. The second process consistent with our results is the generalized reciprocity noted in our introduction, in which individuals that experienced prosocial acts directed towards them by one individual are more likely to act in this way towards other individuals. Our results match 
MPR

Empty Prosocial Self
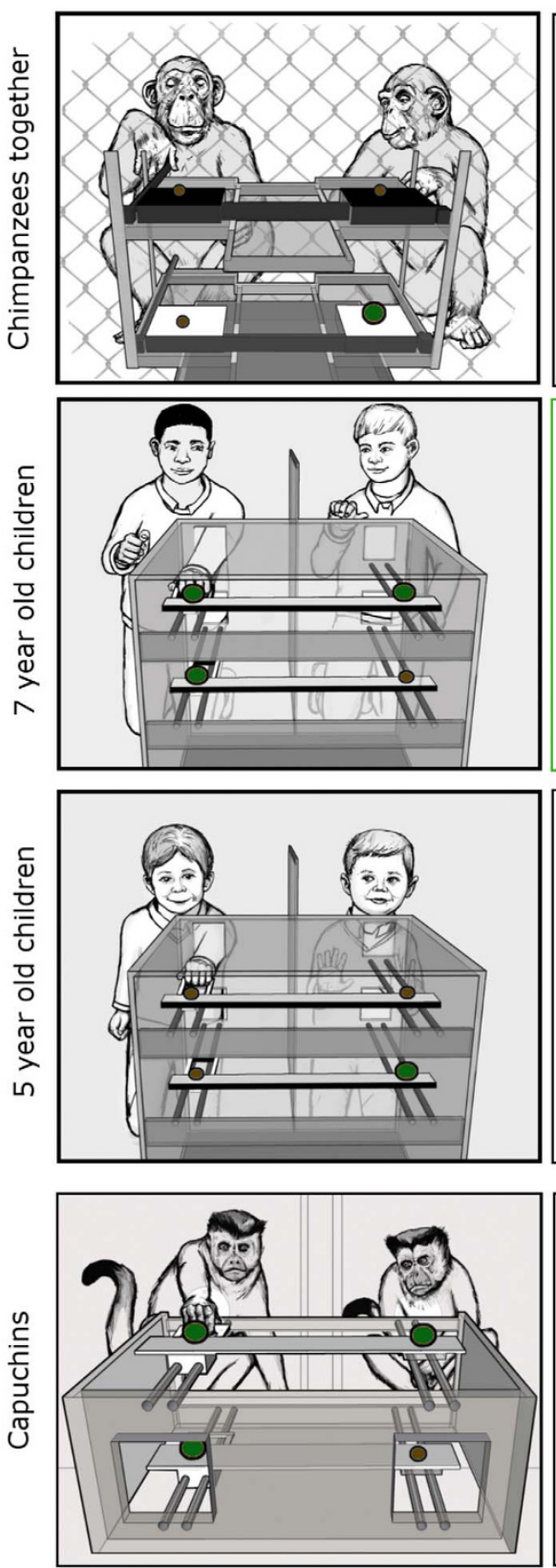
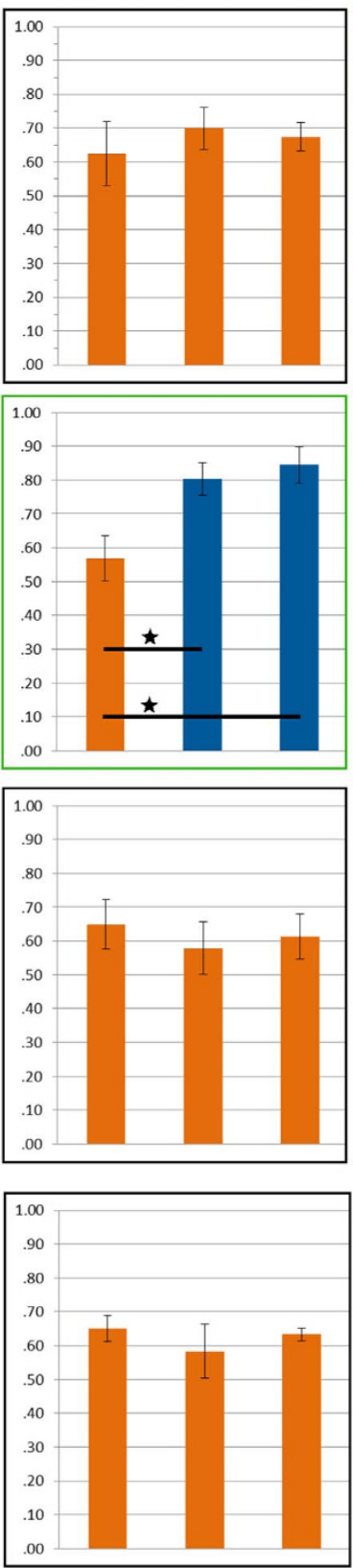

LPR

Empty Prosocial Self
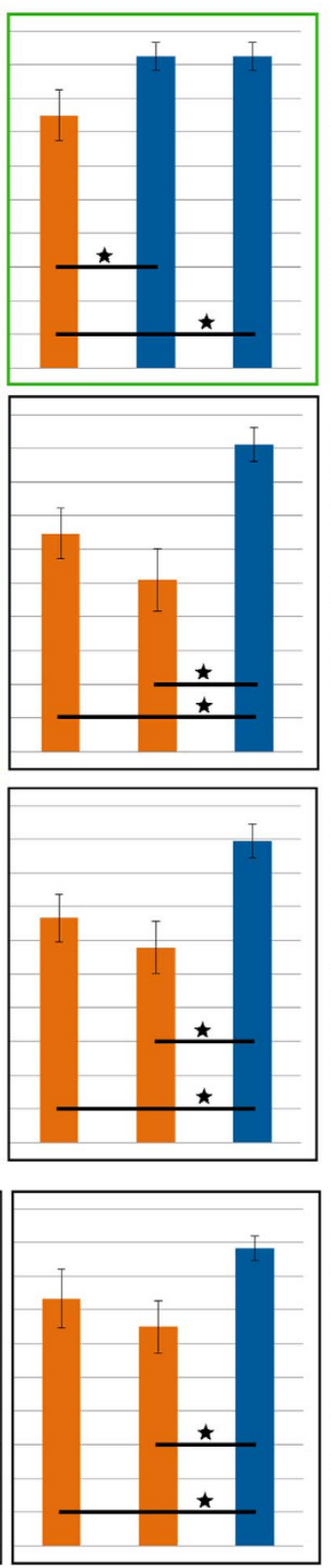

MPR:

$E / S, x 2=0.48, d f=1, p=0.49$

$E / P, x 2=0.32, d f=1, p=0.57$

$P / S, X 2=0.08, d f=1, p=0.78$

LPR:

$E / S, \times 2=11.1, d f=1, p=0.01$

$E / P, X 2=11.1, d f=1, p<0.001$

$P / S, X 2=0.00, d f=1, p=1.00$

MPR:

$E / S, x 2=6.84, d f=1, p=0.009$

$E / P, X 2=14.70, d f=1, p<0.001$

$P / S, x 2=0.373, d f=1, p=0.54$

LPR:

$E / S, x 2=7.38, d f=1, p=0.007$

$E / P, X 2=2.43, d f=1, p=0.12$

$P / S, x 2=9.04, d f=1, p=0.003$

MPR:

$E / S, x 2=0.11, d f=1, p=0.75$

$E / P, x 2=0.75, d f=1, p=0.39$

$\mathrm{P} / \mathrm{S}, \mathrm{X} 2=0.13, \mathrm{df}=1, \mathrm{p}=0.72$

LPR:

$E / S, X 2=6.50, d f=1, p=0.01$

$E / P, X 2=0.66, d f=1, p=0.42$

$P / S, X 2=6.56, d f=1, p=0.01$

MPR:

$E / S, X 2=0.34, d f=1, p=0.56$

$E / P, X 2=1.55, d f=1, p=0.21$

$P / S, X 2=0.62, d f=1, p=0.43$

LPR:

$E / S, x 2=4.40, d f=1, p=0.04$

$E / P, X 2=0.56, d f=1, p=0.46$

$P / S, X 2=30.82, d f=1, p<0.001$

Figure 3 Prosocial responses of chimpanzees, humans and capuchins after the social learning phase. Graphs column 1: More Preferred Rewards (MPR) for the Proposer. Graphs column 2: Less Preferred Rewards (LPR) for the Proposer. Empty (E) = Receiver absent; Prosocial (P) = Receiver present; Self (S) = Self-centered scenario, with Receiver absent and Proposer having access to rewards delivered to Receiver compartment. Blue chart bars = significantly different to Empty condition. Graphs framed in green show a significant prosocial response (Prosocial $>$ Empty). Statistically significant differences are in bold and indicated by stars and statistics given on the right. Drawings by Jason Zampol.

this formulation, since we ensured that any Receiver in the tests of Phase 3 was not the individual who had acted as the prosocial Proposer towards that subject in Phase 2.

Our results make novel and substantial contributions in relation to both of these important processes. First, an explosion of recent studies has documented social learning in children, chimpanzees, capuchins and other primates, but principally in the context of learning about objects and the physical world, rather than about social behavior $^{14,15,20,46-48}$. Conversely, much recent research has addressed a range of prosocial dispositions in children and non-human primates, from helping to food sharing ${ }^{6-11,34,49}$, but their dependence on the influence of others has been only rarely assessed, even in chil- $\mathrm{dren}^{50-53}$, and these studies have not covered the resource-donation which was our focus. The same is true of the handful of studies indicating social influences on social actions, such as reconciliation and affiliation, among non-human primates ${ }^{22,23}$.

Evidence adduced for generalized reciprocity in non-human animals is even rarer, and hitherto has been reported in only a single study of rats $^{54}$. A recent substantial article, demonstrating through modeling that prosociality can evolve through state-dependent, generalized reciprocity ${ }^{28}$, cites only this one empirical study. Our results, consistent with generalized altruism for both children and chimpanzees, provide the first such evidence for non-human primates. Generalized reciprocity can thrive and evolve where helping or dona- 
tion acts as a cue to the level of prosociality in the community one resides in, and although direct reciprocation was not possible within our experimental design, other forms of prosocial donation are likely in the communities we studied, such as grooming services in the case of the non-human primates. These could constitute the broader social contexts in which generalized reciprocal dispositions could be selected for. In the wild, in a chimpanzee community that shows high levels of meat consumption involving large shareable packages (monkeys), as much as $38 \%$ of meat-sharing was classed as 'active' in the sense that the owner was prepared to facilitate the transfer of meat to an individual actively requesting it, by tearing off pieces and offering them ${ }^{55}$. Such social contexts may also include both direct and downstream reciprocity, which Nowak and Roch ${ }^{25}$ showed could facilitate generalized reciprocity (called upstream reciprocity by these authors).

Whether generalized reciprocity is evident in monkeys remains a more open question that has been little addressed. Majolo et al. ${ }^{56}$ found good evidence of direct reciprocity in macaque grooming exchanges, but more patchy evidence for downstream indirect reciprocity and no evidence for generalized reciprocity; likewise, we found no evidence for effects of receiving prosocial donations on capuchin monkey's prosocial acts towards others in our experiments.

Overall, our results confirm that the paradigm used is a demanding one, not eliciting prosocial responses even in all seven year olds, and not in all circumstances, even for those offering prosocial responses. It is important to recognize, however, that other, apparently less demanding forms of prosociality, such as consolation, helping and cooperation may be displayed more commonly in non-human primates $^{6}$ and in children from infancy ${ }^{7,35-37}$.

The results for Phase 3 may be regarded as an additional instance of conditionality: subjects gathered information about the willingness of others to act prosocially. Subsequent to experiencing receipt of multiple prosocial donations of the more preferred of two visible rewards, both chimpanzees and older children who had not displayed a significant degree of prosociality did so. Whether described as social learning or generalized reciprocity, such a response has been shown by theoretical modeling to be evolutionarily viable through positive selection ${ }^{25,26,28}$. Such an effect is predicted to be stronger when participants experience relatively prosocial responses from multiple individuals. While we could expose participants to a maximum of two prosocial models in Phase 2, this will be important to test further. From a social learning perspective, such an effect would be consistent with recent evidence for conformist social transmission, involving adopting the most commonly perceived responses in the population, in both chimpanzees and children ${ }^{14,57-59}$.

\section{Methods}

Apparatus. For the chimpanzees our 'Shelfish' apparatus was adapted from that used previously by Silk, et al. ${ }^{4}$ and Vonk, et al. ${ }^{29}$. The apparatus consisted of two shelves made of clear Lexan $(\mathrm{L} \times \mathrm{W} \times \mathrm{H}: 79 \times 90 \times 45 \mathrm{~cm})$ which were positioned in front of the chimpanzee enclosure, with one side allocated to the Proposer and one to the Receiver. From each position (Proposer or Receiver) four rewards were visible, two located on the upper shelf (one on the Proposer side and one on the Receiver side), and two located directly below on the lower shelf. In their starting position the shelves were positioned out of reach, such that the rewards could not be obtained directly. In order to obtain a reward the Proposer had to pull one of two ropes (which could be accessed from the Proposer's side only) which allowed the food rewards on the selected shelf to be brought towards the Proposer and the Receiver. The Proposer was only allowed to bring one shelf within reach; as one shelf was pulled, the other one automatically retracted.

For the capuchin study the Shelfish apparatus was scaled down appropriately $(\mathrm{L} \times$ $\mathrm{W} \times \mathrm{H}: 50 \times 65 \times 25 \mathrm{~cm}$ ) and modeled on that used previously by

Lakshminarayanan and Santos ${ }^{32}$. During the experiment, the apparatus was placed against two research cubicles that the capuchins are used to sitting in for cognitive testing (one Proposer cubicle, one Receiver cubicle). The shelves were positioned such that the rewards could not be obtained by reaching into the box directly, so to obtain the rewards the Proposer had to reach into the box (through one of two holes in the cubicle door) and pull a handle $(\mathrm{L} \times \mathrm{W}: 11 \times 8 \mathrm{~cm})$ that was attached to the front of each shelf on the Proposer's side.

The Shelfish apparatus used in the human studies was identical in structure to that used for the capuchins but was slightly larger (apparatus: $\mathrm{L} \times \mathrm{W} \times \mathrm{H}$ : $65 \times 65 \times$
$70 \mathrm{~cm}$; shelves: $\mathrm{L} \times \mathrm{W}: 7 \times 57 \mathrm{~cm}$; handles: $\mathrm{L} \times \mathrm{W}: 11 \times 8 \mathrm{~cm})$. In order to create a Proposer and a Receiver compartment equivalent to that of the non-human primates, the area directly in front of the apparatus was divided into two equally-sized compartments by attaching a transparent partition $(\mathrm{L} \times \mathrm{H}: 50 \times 90 \mathrm{~cm})$ perpendicular to the front face of the box.

Participants. Chimpanzee participants were 16 individuals from four groups housed at the Michale E. Keeling Center for Comparative Medicine and Research, UT MD, Anderson Cancer Center, Bastrop, Texas, USA. Twelve individuals (10 females and 2 males) were assigned to the Proposer role, and 4 individuals (all adult females) were assigned to the Receiver role. An additional 2 individuals ( 1 male and 1 female) acted as models for the 4 individuals ( 1 male and 3 females) who were re-tested after the social experience phase. Approval for the study was gained from the UT MD Anderson Institutional Animal Care and Use Committee (IACUC approval number: 07-92-03887) and the Ethics Committee of the University of St Andrews' School of Psychology. Procedures were conducted in accordance with the guidelines of the Association for the Study of Animal Behaviour and UT MD Anderson is fully accredited by the Association for Assessment and Accreditation of Laboratory Animal Care-International.

Capuchin participants were 14 individuals from two groups housed at the 'Living Links to Human Evolution' Research Centre situated within the Royal Zoological Society of Scotland's Edinburgh Zoo, UK. Ten individuals ( 7 males and 3 females) were assigned to the Proposer role, and 4 individuals ( 3 males and 1 female, all juveniles) were assigned to the Receiver role. An additional 4 individuals (all male) acted as models for the 6 individuals ( 3 males and 3 females) who were re-tested after the social experience phase. All procedures were approved by the Royal Zoological Society of Scotland and the Ethics Committee of the University of St Andrews' School of Psychology. Procedures were conducted in accordance with the guidelines of the Association for the Study of Animal Behaviour.

The child study was conducted with 97 individuals recruited from two elementary schools in Scotland, UK. Seventy-nine individuals (34 five-year-olds, mean age 61 months; and 45 seven-year-olds, mean age 92 months) were assigned to the Proposer role, and 18 individuals ( 9 males and 9 females) were assigned to the Receiver role. An additional 22 children ( 12 males and 10 females) acted as models for the 19 five-yearolds ( 11 males and 8 females) and 18 seven-year-olds ( 8 males and 10 females) who were re-tested after the social experience phase.

The adult participants were 51 undergraduate students (mean age 21 years) from a Scottish University who took part in the study for course credit. Forty-one individuals (20 males and 21 females) were assigned to the Proposer Role, and 10 individuals (5 males, 5 females) were assigned to the Receiver role. No social experience phase was conducted with the adult participants due to the high baseline levels of prosociality.

Ethical approval for all of the human research was granted by the School of Life Sciences ethics committee at Heriot Watt University, UK. Informed consent was obtained directly from the adult participants and via the parents of the child participants. All procedures were carried out in accordance with relevant guidelines and regulations.

Procedures. Chimpanzee study. Training phase 1. The aim of the first training phase was to ensure that the participants could distinguish between an MPR (a slice of pineapple) and an LPR (a slice of carrot) on the Proposer's side of the apparatus. This phase additionally served to confirm that the chimpanzees preferred pineapple slices to carrots. During this phase, the participants interacted with the box alone and were allowed to access the Proposer's side only. Every session, of every test always started with familiarization trials in which a single MPR was placed on the Proposer side in one location (top or bottom shelves) and then in the other location. This was meant to reacquaint the participant with the working of the apparatus. The sessions consisted of 10 trials in which an MPR and an LPR were placed on the shelves on both the Proposer and the Receiver's side. The placement of the reward started with the Proposer side, with the top and bottom rewards placed at the same time and followed by the placement of the rewards on the Receiver side (top and bottom shelves at the same time) to attract the attention of the participant to the rewards on the Receiver side of the apparatus (see supplementary videos of each experiment for more details regarding the setup). The placement of the rewards on the top and bottom shelves was counterbalanced, at this stage and in following phases. Participants were trained using this procedure until they made at least 16 retrievals of the MPR from the Proposer's side in two consecutive sessions (out of 20 trials; at least $80 \%$ success).

Training phase 2 . The second training phase aimed to ensure that the participants were capable of attending to the value of the rewards on both the Proposer's and Receiver's sides of the apparatus. This was achieved by allowing the chimpanzees to access the rewards from both the Proposer's and the Receiver's side. The rewards available on the Proposer's side were always identical (two LPRs or two MPRs), whereas the rewards on the Receiver's side always differed (one LPR and one MPR). In order to obtain the maximum payoff, the participants had to attend to the value of the rewards on the Receiver's side. Participants were trained using this procedure until they made at least 16 retrievals of the MPR from the Receiver's side in two consecutive sessions (out of 20 trials; at least $80 \%$ success).

Testing Phase 1-Prosocial Baseline. The first testing phase occurred immediately after the training phase and comprised three conditions: two control conditions (empty control and self-centered control), and a prosocial test condition. Each participant performed two sessions of 10 trials of each condition, with order of conditions randomized. This was followed by another set of two sessions of each condition re-ordered pseudo-randomly so that the last session of the first test was not identical 
Testing phase 2: absence of LPR on the Receiver side. The purpose of the second

to the first session of the second test (in other words, individuals were never exposed to 40 trials of the same condition). Each participant therefore received a total of 120 trials (40 prosocial, 40 empty and 40 self-centered). In both control conditions the participants interacted with the apparatus individually. The difference between the two conditions was that a barrier (partition or wall) was present in the empty control condition, allowing the participant to access the rewards on the Proposer's side only, whereas the barrier was removed in the self-centered control condition, allowing the participants access to both the Proposer and Receiver sides.

In the prosocial test condition Proposers were tested with a group mate in the Receiver compartment. In the together condition, Proposer and Receiver were in the same room, with no barrier between them. To make sure that they remained in their respective roles we trained them to receive the rewards from their position and removed the apparatus from reach if one or the other tried to reach for the reward on the other side.

In each of the three conditions, the trials comprised two different pay-off structures presented in a pseudo-randomly counterbalanced sequence within each session of 10 trials (the number of successive trials of the same type did not exceed three): five MPR trials and five LPR trials. In the LPR trials (LPR \& LPR versus LPR \& MPR) and the MPR trials (MPR \& MPR versus LPR \& MPR), the outcome for the Proposer side was identical irrespective of which shelf they chose to pull. However, the choice of shelf influenced the outcome for the Receiver, with the Proposer having the option of delivering either a MPR or a LPR to the Receiver's side.

Testing phase 2-Social experience. Once the initial level of prosociality had been established, the participants entered the social experience phase in which they were placed in the Receiver compartment, with a new 'prosocial' individual on the Proposer side. In order to ensure that the prosocial model would deliver the MPR on every trial, the shelf with the LPR on the Receiver's side was locked in place (by securing the shelf to the frame of the apparatus) so that it could not be brought forward. The participants completed 4 sessions ( 40 trials in total) in which a pretrained model was in the role of Proposer and the test participant was in the role of Receiver. The order of the trials was determined using the same pseudo-randomization procedure as in Testing phase 1 .

Testing phase 3-Prosocial Re-test. On completion of the social experience phase, the test participants were re-positioned on the Proposer's side of the box and re-tested with the same Receiver from phase 1 . The participants were again tested in the three different conditions (empty, self-centered and prosocial) to determine whether any increase in prosociality was a result of being exposed to the prosocial model, rather than the participants simply pulling the highest value shelf more often. Each block of trials was presented in an order determined according to the same procedure as before, for a total of 60 trials (two consecutive sessions of 10 trials per condition).

Testing phases two and three were performed with the chimpanzees in the together condition and not in the separate condition because the design of the housing facility prevented us from completing these two phases with the latter chimpanzees.

Capuchin study. The procedure for the capuchin study mirrored that of the chimpanzees almost exactly. As with the chimpanzees, each Proposer was required to meet criterion ( $80 \%$ success) in both training phases (using papaya as MPR and pellets as LPR), before completing 120 trials ( 40 prosocial, 40 empty and 40 self-centered) in the first experimental phase (10 trials per session). Those individuals who proceeded to the social experience phase received the MPR from either a single model, or from two models, for a total of 40 trials, before being re-tested with their original Receiver (60 trials in total, 20 prosocial, 20 empty and 20 self-centered). Every session, of every test also started with familiarization trials in which a single MPR was successively placed in one of the four possible locations (top or bottom shelves on the Proposer or Receiver side). This was meant to reacquaint the participant with the working of the apparatus.

Study on the conditional prosocial response of capuchin monkeys: We decided to explore three factors that could potentially explain the origin of the variability in prosocial tendency observed in capuchin monkeys. (i) A large difference between the MPR and LPR could limit the tendency of Proposers to give the MPR to Receivers (ii) On the contrary, the difference in quality between the MPR and LPR on the Receiver's side may not be well recognized (iii) Finally, it is possible that the presence of two MPR facing the Proposer distracted him/her from the Receiver's side of the apparatus and this masked the effect that could be revealed if the food was not visible.

These experiments were conducted more than 6 months after the end of the first study, from June 2012 to August 2012. Eight capuchins that were tested in the previous study took part with their original Receivers.

Training phase: One training session of 10 trials was used to enable the capuchins to accustom themselves to the apparatus once more. A reward was randomly placed in one of the 4 possible locations with the partition open. We randomly permutated the sequence of four locations three times to obtain a succession of 12 locations that we then cut to 10 (each location was therefore sampled at least twice). The goal was to ensure that the participants could remember which shelf they needed to pull to get the reward. The following test phases started with 4 familiarization trials, as in the previous study. The participants completed 2 sessions of 10 trials in in each testing phase.

Testing phase 1: reduction of the difference between MPR and LPR. The purpose of this first phase was to see if a reduction in the difference between the MPR and LPR would influence the level of prosociality. Capuchins prefer raisins to pellets and papaya to raisins, so we decided to use raisins as a LPR instead of pellets and kept all other procedures and settings identical to the prosocial test phase. testing phase was to evaluate the importance of the presence of the LPR on the Receiver side. The procedure was identical to the previous phase except that the LPR on the Receiver side was removed. The Proposer therefore had the choice between raisin/nothing vs. raisin/papaya in the LPR trials, and papaya/papaya vs. papaya/ nothing in the MPR trials.

Testing phase 3: Capuchins delayed condition. Before performing testing phase 3, Proposers were trained to experience a delay in receiving their reward. First during 2 sessions ( 20 trials), the partition was open and a MPR was available to the participant on either the top or the bottom shelf on the Receiver side. There was no reward on the Proposer side, however, immediately after having pulled one shelf (whether or not it bore a reward), the experimenter handed a MPR to the participant on the Proposer side. The participant therefore could not access the MPR on the Proposer side directly and experienced a short delay in receiving the reward from the experimenter. After these 20 trials, the partition was closed again and a MPR was placed in the now inaccessible Receiver cubicle. As soon as the participant pulled one of the two shelves, the experimenter rewarded the participant with a MPR. This training was performed for 2 sessions of 10 trials. Once the participants had completed training, they were paired with their original Receiver (partition closed) for two sessions of 10 trials and only one MPR was placed on the Receiver's side. The Proposers were rewarded by a MPR every time they pulled one of the shelves.

Post-test: After completion of the three testing phases, the participants performed two control conditions equivalent to the empty and self-centered controls described previously. In the first 2 control sessions (the delayed empty control) the partition was closed, the Receiver was absent and a MPR was placed on the Receiver's side. The following 2 control sessions (the delayed self-centered control) were identical to those presented previously with the exception that the partition was open, allowing the participant to access the reward on the Receiver's side. During all these trials, the participants were rewarded for pulling one of the two shelves with a MPR.

Child Study. The procedure for the child study aimed to replicate that for the nonhuman primates as closely as possible, whilst acknowledging the practicalities of working with children. These considerations led to several minor procedural changes for the child participants. Throughout each phase, the food rewards were replaced with a colorful sticker (MPR) and a plain white sticker (LPR). Stickers were used as the rewards in order to comply with school and parental preferences. The use of stickers as rewards is commonplace in developmental psychology and previous studies have shown that children are highly motivated by stickers. We are therefore confident that the rewards used for each species, although different, were motivating in each case.

The children were asked to complete fewer trials and participate in fewer sessions than the non-human primates, as is common in comparative research of this nature. The training and baseline testing phase were conducted in a single 30-minute session, with the social experience and prosocial re-test occurring in a single 30-minute session one week later. Following the training phase (criterion for each phase was two consecutive successes in a maximum of eight trials given that the working of the apparatus is transparent for children), the children were presented with a total of 18 trials divided into 3 counter-balanced blocks ( 6 empty, 6 self-centered, and 6 prosocial). Those individuals who proceeded to the social experience phase (delivered $<5$ MPRs from a maximum of 6 at baseline) assumed the role of Receiver with two different prosocial models for a total of 8 trials. In order to ensure that the child models appeared to be delivering the MPR through choice, rather than being forced to do so, we pre-trained the child models to deliver the rewards with both shelves unlocked. On completion of the social experience phase, the participants were re-tested in the phase 1 configuration with the order of the three conditions re-randomized.

Adult study. The procedure of the adult study was identical in every respect to the child study with the exception that the rewards were coins (MPR $=50$ UK pence; LPR $=20$ UK pence) rather than stickers. Due to the high baseline level of prosociality we documented for adults, no social experience phase was conducted with these adult participants.

Statistical procedures. Given that our response variable was binary ('prosocial' or 'not prosocial' choice), we used a binomial generalized estimating equation model ${ }^{60}$ (GEE), with logit link function, to analyze the data (see Supplementary information for additional details). The GEE approach represents an extension of Generalized Linear Models (GLM) for the analysis of repeated measurement data and other nonindependent data. Compared to Generalized Linear Mixed Models (GLMM), the focus of GEE is on estimating the population effects (marginal model) rather than the regression parameters (conditional model) that would enable prediction of the effect for a given individual. In our study, non-independence could arise from different levels (repeated measurements, several group levels such as school and classroom for instance, etc.) and the use of GEE allowed us to use the same statistical technique for each species and study, while still taking into account different levels of nonindependence. However, given that GEE and GLMM are recently developed statistical methods that are still relatively new to the field ${ }^{61,62}$, we also conducted our analyses using GLMM. Since we obtained qualitatively similar results with GEE and GLMM, we report only the results from the GEE.

All the statistical comparisons performed were planned and follow logically from the design of the experiments, so we report exact, uncorrected, p-values.

1. Henrich, J. et al. Foundations of human sociality: economic experiments and ethnographic evidence from fifteen small-scale societies. (Oxford University Press, Oxford, 2004). 
2. Alexander, M. \& Christia, F. Context Modularity of Human Altruism. Science 334, 1392-1394, doi:10.1126/science.1202599 (2011).

3. Fehr, E. \& Fischbacher, U. The nature of human altruism. Nature 425, 785-791 (2003).

4. Silk, J. et al. Chimpanzees are indifferent to the welfare of unrelated group members. Nature 437, 1357-1359 (2005)

5. Jensen, K., Hare, B., Call, J. \& Tomasello, M. What's in it for me? Self-regard precludes altruism and spite in chimpanzees. Proc. R. Soc. Lond. B. 273, 1013-1021, doi:10.1098/rspb.2005.3417 (2006).

6. de Waal, F. B. M. \& Suchak, M. Prosocial primates: selfish and unselfish motivations. Philos. Trans. R. Soc. Lond. B. Biol. Sci. 365, 2711-2722, doi:10.1098/ rstb.2010.0119 (2010).

7. Warneken, F. \& Tomasello, M. Altruistic Helping in Human Infants and Young Chimpanzees. Science 311, 1301-1303, doi:10.1126/science.1121448 (2006).

8. Melis, A. P. et al. Chimpanzees help conspecifics obtain food and non-food items. Proc. R. Soc. Lond. B. 278, 1405-1413, doi:10.1098/rspb.2010.1735 (2011).

9. Warneken, F., Hare, B., Melis, A. P., Hanus, D. \& Tomasello, M. Spontaneous Altruism by Chimpanzees and Young Children. PLoS Biol. 5, 1414-1420, doi:10.1371/journal.pbio.0050184 (2007).

10. LoBue, V., Nishida, T., Chiong, C., DeLoache, J. S. \& Haidt, J. When Getting Something Good is Bad: Even Three-year-olds React to Inequality. Soc. Dev. 20, 154-170, doi:10.1111/j.1467-9507.2009.00560.x (2011).

11. Subiaul, F., Cantlon, J. F., Holloway, R. L. \& Terrace, H. S. Cognitive Imitation in Rhesus Macaques. Science 305, 407-410, doi:10.1126/science.1099136 (2004).

12. Fehr, E., Bernhard, H. \& Rockenbach, B. Egalitarianism in young children. Nature 454, 1079-1083 (2008)

13. House, B. R., Henrich, J., Brosnan, S. F. \& Silk, J. B. The ontogeny of human prosociality: behavioral experiments with children aged 3 to 8. Evol. Hum. Behav. 33, 291-308, doi:10.1016/j.evolhumbehav.2011.10.007 (2012).

14. Haun, D. B. M., Rekers, Y. \& Tomasello, M. Majority-Biased Transmission in Chimpanzees and Human Children, but Not Orangutans. Curr. Biol. 22, 727-731 (2012).

15. van de Waal, E., Borgeaud, C. \& Whiten, A. Potent social learning and conformity shape a wild primate's foraging decisions. Science 340, 483-485 (2013).

16. Price, E. E., Lambeth, S. P., Schapiro, S. J. \& Whiten, A. A potent effect of observational learning on chimpanzee tool construction. Proc. R. Soc. Lond. B. 276, 1471-2954, doi:10.1098/rspb.2009.0640 (2009).

17. Whiten, A., Hinde, R. A., Stringer, C. B. \& Laland, K. N. Culture Evolves. Philos. Trans. R. Soc. Lond. B. Biol. Sci. 366, 938-948 (2011).

18. Kamilar, J. M. \& Atkinson, Q. D. Cultural assemblages show nested structure in humans and chimpanzees but not orangutans. Proc. Natl. Acad. Sci. U.S.A. 111, 111-115, doi:10.1073/pnas.1313318110 (2014).

19. Whiten, A. et al. Transmission of multiple traditions within and between chimpanzee groups. Curr. Biol. 17, 1038-1043 (2007).

20. Whiten, A., Horner, V. \& de Waal, F. B. Conformity to cultural norms of tool use in chimpanzees. Nature 437, 737-740 (2005).

21. Hopper, L. M., Schapiro, S. J., Lambeth, S. P. \& Brosnan, S. F. Chimpanzees' socially maintained food preferences indicate both conservatism and conformity. Anim. Behav. 81, 1195-1202, doi:10.1016/j.anbehav.2011.03.002 (2011).

22. Sapolsky, R. M. \& Share, L. J. A pacific culture among wild baboons: Its emergence and transmission. PLoS Biol. 2, 534-541 (2004).

23. de Waal, F. B. M. \& Johanowicz, D. L. Modification of Reconciliation Behavior Through Social Experience: An Experiment with Two Macaque Species. Child Dev. 64, 897-908 (1993).

24. Brosnan, S. F. Justice- and fairness-related behaviors in nonhuman primates. Proc. Natl. Acad. Sci. U.S.A. 110, 10416-10423, doi:10.1073/pnas.1301194110 (2013).

25. Nowak, M. A. \& Roch, S. Upstream reciprocity and the evolution of gratitude. Proc. R. Soc. Lond. B. 274, 605-610, doi:10.1098/rspb.2006.0125 (2007).

26. Pfeiffer, T., Rutte, C., Killingback, T., Taborsky, M. \& Bonhoeffer, S. Evolution of cooperation by generalized reciprocity. Proc. R. Soc. Lond. B. 272, 1115-1120, doi:10.1098/rspb.2004.2988 (2005).

27. Rutte, C. \& Pfeiffer, T. Evolution of reciprocal altruism by copying observed behaviour. Curr. Sci. 97, 1573-1578 (2009).

28. Bartal, I. B.-A., Decety, J. \& Mason, P. Empathy and Pro-Social Behavior in Rats. Science 334, 1427-1430, doi:10.1126/science.1210789 (2011).

29. Vonk, J.et al. Chimpanzees do not take advantage of very low cost opportunities to deliver food to unrelated group members. Anim. Behav. 75, 1757-1770, doi: 10.1016/j.anbehav.2007.09.036 (2008)

30. Yamamoto, S. \& Tanaka, M. The influence of kin relationship and reciprocal context on chimpanzees' other-regarding preferences. Anim. Behav. 79, 595-602, doi: 10.1016/j.anbehav.2009.11.034 (2010).

31. Horner, V., Carter, J. D., Suchak, M. \& de Waal, F. B. M. Spontaneous prosocial choice by chimpanzees. Proc. Natl. Acad. Sci. U.S.A. 108, 13847-13851, doi: 10.1073/pnas.1111088108 (2011).

32. Lakshminarayanan, V. R. \& Santos, L. R. Capuchin monkeys are sensitive to others' welfare. Curr. Biol. 18, R999-R1000 (2008).

33. Cronin, K. A. Prosocial behaviour in animals: the influence of social relationships, communication and rewards. Anim. Behav. 84, 1085-1093, doi: 10.1016/ j.anbehav.2012.08.009 (2012).

34. Burkart, J. M., Fehr, E., Efferson, C. \& van Schaik, C. P. Other-regarding preferences in a non-human primate: Common marmosets provision food altruistically. Proc. Natl. Acad. Sci. U.S.A. 104, 19762-19766, doi: 10.1073/ pnas.0710310104 (2007).

35. Svetlova, M., Nichols, S. R. \& Brownell, C. A. Toddlers' Prosocial Behavior: From Instrumental to Empathic to Altruistic Helping. Child Dev. 81, 1814-1827, doi: 10.1111/j.1467-8624.2010.01512.x (2010).

36. Brownell, C. A., Svetlova, M. \& Nichols, S. To Share or Not to Share: When Do Toddlers Respond to Another's Needs? Infancy 14, 117-130 (2009).

37. Dean, L. G., Kendal, R. L., Schapiro, S. J., Thierry, B. \& Laland, K. N. Identification of the Social and Cognitive Processes Underlying Human Cumulative Culture. Science 335, 1114-1118, doi: 10.1126/science.1213969 (2012).

38. Blake, P. R. \& McAuliffe, K. "I had so much it didn't seem fair": Eight-year-olds reject two forms of inequity. Cognition 120, 215-224, doi: 10.1016/ j.cognition.2011.04.006 (2011).

39. Brosnan, S. F., Talbot, C., Ahlgren, M., Lambeth, S. P. \& Schapiro, S. J. Mechanisms underlying responses to inequitable outcomes in chimpanzees, $\mathrm{Pan}$ troglodytes. Anim. Behav. 79, 1229-1237, doi: 10.1016/j.anbehav.2010.02.019 (2010).

40. Brosnan, S. F. A hypothesis of the co-evolution of cooperation and responses to inequity. FNINS 5, 1-12 (2011).

41. Takagishi, H., Kameshima, S., Schug, J., Koizumi, M. \& Yamagishi, T. Theory of mind enhances preference for fairness. J. Exp. Child. Psychol. 105, 130-137, doi: 10.1016/j.jecp.2009.09.005 (2010).

42. McAuliffe, K., Blake, P. R., Kim, G., Wrangham, R. W. \& Warneken, F. Social Influences on Inequity Aversion in Children. PLoS ONE 8, 1-11, doi: 10.1371/ journal.pone.0080966 (2013).

43. Brosnan, S. F. \& de Waal, F. B. M. Monkeys reject unequal pay. Nature 425, 297-299 (2003)

44. de Waal, F., Leimgruber, K. \& Greenberg, A. R. Giving is self-rewarding for monkeys. Proc. Natl. Acad. Sci. U.S.A. 105, 13685-13689 (2008).

45. Heyes, C. M. Social learning in animals: categories and mechanisms. Biol. Rev. 69, 207-231, doi:10.1111/j.1469-185X.1994.tb01506.x (1994).

46. Claidière, N., Messer Emily, J. E., Hoppitt, W. \& Whiten, A. Diffusion Dynamics of Socially Learned Foraging Techniques in Squirrel Monkeys. Curr. Biol. 23, 1251-1255 (2013).

47. Whiten, A. The scope of culture in chimpanzees, humans and ancestral apes. Philos. Trans. R. Soc. Lond. B. Biol. Sci. 366, 997-1007, doi: 10.1098/ rstb.2010.0334 (2011).

48. Frith, C. D. \& Frith, U. Mechanisms of Social Cognition. Annu. Rev. Psychol. 63, 287-313, doi: 10.1146/annurev-psych-120710-100449 (2012).

49. Silk, J. B. \& House, B. R. Evolutionary foundations of human prosocial sentiments Proc. Natl. Acad. Sci. U.S.A. 108, 10910-10917, doi: 10.1073/pnas.1100305108 (2011).

50. Harbaugh, W. T., Krause, K. \& Vesterlund, L. Learning to bargain. J. Econ. Psychol. 28, 127-142, doi: 10.1016/j.joep.2006.03.005 (2007).

51. Williamson, R. A., Donohue, M. R. \& Tully, E. C. Learning how to help others Two-year-olds' social learning of a prosocial act. J. Exp. Child. Psychol. 114, 543-550, doi: 10.1016/j.jecp.2012.11.004 (2013).

52. Carpenter, M., Uebel, J. \& Tomasello, M. Being Mimicked Increases Prosocial Behavior in 18-Month-Old Infants. Child Dev. 84, 1511-1518, doi: 10.1111/ cdev.12083 (2013).

53. Hamann, K., Warneken, F., Greenberg, J. R. \& Tomasello, M. Collaboration encourages equal sharing in children but not in chimpanzees. Nature 476, 328-331, doi: 10.1038/nature10278 (2011)

54. Rutte, C. \& Taborsky, M. Generalized Reciprocity in Rats. PLoS Biol. 5, 1421-1425 (2007).

55. Boesch, C. \& Boesch, H. Hunting behavior of wild chimpanzees in the Tai National Park. Am. J. Phys. Anthropol. 78, 547-573, doi: 10.1002/ ajpa.1330780410 (1989)

56. Majolo, B., Schino, G. \& Aureli, F. The relative prevalence of direct, indirect and generalized reciprocity in macaque grooming exchanges. Anim. Behav. 83, 763-771, doi: 10.1016/j.anbehav.2011.12.026 (2012).

57. Claidière, N. \& Whiten, A. Integrating the study of conformity and culture in humans and nonhuman animals. Psych. Bull. 138, 126-145, doi: 10.1037/ a0025868 (2012)

58. Corriveau, K. \& Harris, P. Preschoolers (sometimes) defer to the majority in making simple perceptual judgments. Dev. Psych. 46, 437-445 (2010).

59. Corriveau, K., Fusaro, M. \& Harris, P. Going with the flow: Preschoolers prefer nondissenters as informants. Psychol. Sci. 20, 372-377 (2009).

60. Liang, K.-Y. \& Zeger, S. L. Longitudinal data analysis using generalized linear models. Biometrika 73, 13-22, doi:10.1093/biomet/73.1.13 (1986).

61. Heagerty, P. J. \& Zeger, S. L. Marginalized multilevel models and likelihood inference. Stat. Sci. 15, 1-26 (2000).

62. Lee, Y. \& Nelder, J. A. Conditional and Marginal Models: Another View. Stat. Sci. 19, 219-238 (2004)

\section{Acknowledgments}

We thank the parents, children and teaching staff (especially Gill Gardner and Kirstie Miller) at Longniddry and Granton Primary Schools, Dee Masters, and the primate care staff at the Living Links Centre and the Michale E. Keeling Center for Comparative Medicine and Research Center for their support, Jason Zampol for illustrations, and Andy 
Burnley for apparatus construction. The chimpanzees at the Keeling Center were supported by NIH Cooperative Agreement U42 OD-011197. The project was supported by a grant from the John Templeton Foundation (Ref 20721) to AW. The funders had no role in study design, data collection and analysis, decision to publish, or preparation of the manuscript.

\section{Author contributions}

A.W., N.C. and N.McG. designed and supervised the experimental program. N.C., N.McG., E.J.E.M. and M.C.M. conducted the experiments. S.F.B., L.M.H., S.P.L. and S.J.S. provided essential logistical support and guidance at the Bastrop chimpanzee facility. N.C. and N.McG. conducted statistical analyses. N.C., A.W. and N.McG. wrote the paper. All authors discussed the results and commented on the manuscript.

\section{Additional information}

Supplementary information accompanies this paper at http://www.nature.com/ scientificreports

Competing financial interests: The authors declare no competing financial interests. How to cite this article: Claidière, N. et al. Selective and contagious prosocial resource donation in capuchin monkeys, chimpanzees and humans. Sci. Rep. 5, 7631; DOI:10.1038/ srep07631 (2015).

This work is licensed under a Creative Commons Attribution-NonCommercialNoDerivs 4.0 International License. The images or other third party material in this article are included in the article's Creative Commons license, unless indicated otherwise in the credit line; if the material is not included under the Creative Commons license, users will need to obtain permission from the license holder in order to reproduce the material. To view a copy of this license, visit http:// creativecommons.org/licenses/by-nc-nd/4.0/ 